\title{
Livelihood Security of Farm Households in Eastern Dry Zone of Karnataka - An Economic Analysis
}

\author{
A. P. Bhavya ${ }^{*}$, B. C Ashwini and K. B. Umesh \\ Department of agricultural economics, UAS, GKVK, Bengaluru-65, India \\ *Corresponding author
}

\section{A B S T R A C T}

\section{Keywords}

Farming system, Livelihood security, Food security, Food consumption

\section{Article Info}

Accepted:

24 July 2020

Available Online:

10 August 2020
The present research work makes an effort to understand the livelihood security of farmers adopting various farming systems like Crop + Sheep, Crop + Dairy, Crop + Dairy + Sericulture and Crop + Dairy + Horticulture in EDZ of Karnataka. The livelihood index has been developed for farm households under different sub components like Economics, food, health, education, habitat, social participation and composite index. The composite livelihood security of $\mathrm{C}+\mathrm{D}+\mathrm{H}$ farming system was 0.49 higher than the other farming systems. In case of economic security, $\mathrm{C}+\mathrm{D}+\mathrm{H}$ farming system households had highest index (0.42) than the other farming systems. The dairy and sericulture components contribute highest to the total income in the existing farming system. Hence, the addition of enterprises like dairy, sericulture, sheep, horticulture, which are suitable to the zone can add to the income, besides enhancing the nutritional and livelihood security of the farm households.

\section{Introduction}

Indian agriculture is characterized with huge labour force, rank first with the world's highest net cropped area and rank second in farm output in the world. However, agricultural growth in India is decelerating every year $(12.89 \%$ in 1975 to $2.1 \%$ in 2017) (www.indianstat.com) and at the same time the government is trying hard to double the farm income. Hence, there is a need to integrating different farming systems to provide better livelihood is the need of hour to enhance the farm income (Harishkumar et al., 2016). Farming system is the result of interaction among several interdependent components like crops, dairy, poultry, sericulture, piggery, sheep, goat, fisheries, bee-keeping etc. (Norman, 1978). Livelihood refers to adequate stock and flow of food and cash with an individual to meet their basic needs and livelihood security means secured ownership of, access to resources and income earning activities, including reserves and assets to offset risk, ease shocks and meet contingencies (Shyamli et al., 2013; Ijarotimi and Oyeneyin, 2005). The concept of household livelihood security allows for a more comprehensive understanding of poverty, malnutrition, dynamic and complex strategies that the people use for survival. With this background, effort is made to 
analyze livelihood security of farmers who practice various farming systems like crop + sheep, crop +dairy, crop + dairy + sericulture and crop + dairy + horticulture in the Eastern dry zone of Karnataka.

\section{Materials and Methods}

In present study, a random and purposive sampling procedure has been adopted for the selection of districts, taluks, villages and farmers. In the first stage, purposively Kolar district representing the eastern dry zone of Karnataka was selected based on the rainfed farming with ground water irrigation system followed in agriculture. In the second stage, based on the major farming systems practiced in the district, three taluks were selected Viz, Bangarpet taluk representing Crop + Sheep (C + Sh) faming system, Kolar for Crop + Dairy + Sericulture $(C+D+S)$ farming system, and Mulbagal representing both Crop+ Dairy $(\mathrm{C}+$ D) and Crop + Dairy + Horticulture $(\mathrm{C}+\mathrm{D}+$ $\mathrm{H}$ )farming systems. In the third stage, three villages from each taluk were randomly selected, and for each village 10 samples were collected make a total sample size of 120 .

The data were collected from primary and secondary sources. Personal interview method was followed to collect the primary data using pre-tested schedule. The collected data was analyzed using conventional methods like frequency, averages and proportion. Livelihood security index was developed to estimate the livelihood security status of the different farm households.

\section{Livelihood security index}

Conceptual frame work The household livelihood security index (HLS) uses a balanced weighted average approach with a large number of indicators, where each indicator is assumed to contribute equally to the overall index (Venu et al., 2018) The indicators are grouped into different domains representing the security areas such as economic, nutrition, health, education, habitat and social-participation.

In this paper, we have considered the Maxwell and Frankenberger model has been considered to access the household livelihood security. The major component of livelihood security include (Sakamma, 2013).

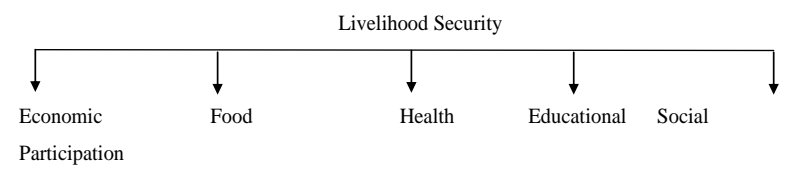

Economic security: includes total annual income earned by the farmers, value of farm land, value of livestock, value of non land farm assets and household savings.

Food security: includes quantity of food consumption and its expenditure.

Education security: consists of accessibility and availability of educational institutions in the study area.

Health security: consists of expenditure on health problems, accessibility and availability of health centers.

Habitat security: includes type of house (Pakka, Semi pakka, and Kaccha house), accessibility and availability of drinking water, toilet facility and cooking gas connection.

Social-participation security: consists of number of members participating in SHG, Milk cooperatives, Gram panchayath, Taluk panchayath and ownership of TV and Mobile phones.

Since each indicator is measured on a different scale, indicators are standardized following the approach adopted in measuring 'Life Expectancy' in Human Development 
Reports (Akter and Rahman, 2012).

For example, a standardised indicator $j$ is given by:

Zind $\mathrm{j}=\frac{\text { indicator } j-\min j}{\max j-\min j}$

Where minimum and maximum values of the indicators are from the same components to which the $\mathrm{j}$ is considered. Once each indicator representing a particular livelihood security domain is standardised, then the relevant household livelihood security index for the particular domain is constructed by averaging the standardized indicators:

$\mathrm{HLS}_{\mathrm{j}}=\sum_{I=1}^{j} z^{i n d j} / \mathrm{j}$

Where: $j$ is the number of indicators used to construct the index.

The Composite overall Livelihood Security (CLS) index for the household is constructed by using the formula.

$\mathrm{CLS}=\sum_{i=1}^{n}$ wiHLSi $/ \sum_{i=1}^{n} w i$

Where,

w - Indicates the weights determined by the number of indicators used to construct each HLS index. A weight varies among households, because of the variation in the number of indicators at the household level.

\section{Results and Discussion}

\section{Economic security}

Table 1 indicates the land holding pattern of the farm households in Kolar district. The average land holding was found to be higher in $\mathrm{C}+\mathrm{D}+\mathrm{S}$ farming system followed by $\mathrm{C}+$ $\mathrm{D}+\mathrm{H}$ farming system. Average land holding of rainfed farming of all the farming systems were more than irrigated farming systems. Kolar district comes under the Eastern dry zone of Karnataka where water supplementation from upper catchment is not available so farmers are highly reliable on ground water and rainfall for agriculture production.

Table 2 indicates the ownership of fixed assets under major farming systems in Kolar district. The value of land per farm was found to be highest in $\mathrm{C}+\mathrm{D}+\mathrm{S}$ farming system followed by $\mathrm{C}+\mathrm{D}+\mathrm{H}$ farming system. This is due to location of the farm land is very near to the city centers and average size of the farm (1.69 and $1.5 \mathrm{ha})$ and interestingly contribution of value of non land farm asset (14\%) was found to be highest in C + D + H farming system in Kolar district.

In the Table 3 Indicate the net income of farm households from various sources in Kolar district. $\mathrm{C}+\mathrm{D}+\mathrm{S}$ farmers used to get a maximum net annual income of Rs. 376453 of which 57.72 per cent was sourced from sericulture and 36.19 per cent from livestock and only four per cent from crop due the lower income crops grown by the farmers. Here it is notice that livestock contribution to the total income is more than 30 per cent in all the farming systems and has become source of sustainable income for the farmers (Sachin, 2012, and Zainab, 2010).

\section{Food Security}

Quantity of food consumption and expenditure pattern of farm households of different farming systems in the study area is presented in Table 3. It clearly shows that dietary pattern of households in all groups was mainly cereal based. Average consumption of cereals was estimated higher in $\mathrm{C}+\mathrm{D}+\mathrm{S}(86 \mathrm{~kg} /$ family/month $)$ and lower in $\mathrm{C}+\mathrm{D}$ (54 kg/family/month). Average 
consumption of pulses was low in all the farming systems Compared with the ICMR recommendation ( $80 \mathrm{gm} /$ day) in the districts. Average consumption of vegetables, fruits, milk, edible oil and sugar was higher in $\mathrm{C}+\mathrm{D}$ $+\mathrm{H}$ based farm households and lower in $\mathrm{C}+$ Sh farm households which has to be correlated to level of farm income and average family size in both the district.

Average monthly expenditure on meat and egg was found higher in $\mathrm{C}+\mathrm{D}+\mathrm{S}$ based farming system in Kolar district and Monthly consumption expenditure was found higher in C + D + S (Rs.5164) followed by C + Sh (Rs. 4885) based farming system it is due average number of family member per farm, consumption pattern and net annual income of sample respondent. This result is contradictory with the findings of Harishkunmar (2012).

\section{Health Security}

Health is one of the important factors in livelihood security of households. Security of farm households in terms of health is defined by way of availability and accessibility of health services like primary health center, 24 hours facility, Specialty hospital, and Yashaswini card.

Table.1 Land holding pattern of farm households in Kolar District (Hectare per farm)

\begin{tabular}{|l|r|r|r|r|r|}
\hline Type of Land holding & $\begin{array}{l}\text { C+ Sh } \\
(\mathbf{n = 3 0 )}\end{array}$ & $\begin{array}{l}\text { C+ D + S } \\
(\mathbf{n = 3 0 )}\end{array}$ & $\begin{array}{l}\text { C+ D } \\
(\mathbf{n = 3 0})\end{array}$ & $\begin{array}{l}\text { C+ D + H } \\
(\mathbf{n = 3 0})\end{array}$ & F value \\
\hline Irrigated & 0.12 & 0.57 & 0.22 & 0.38 & $11.38^{* * *}$ \\
\hline Rainfed & $(10.25)$ & $(33.72)$ & $(16.66)$ & $(25.33)$ & \\
\hline Present Fallow land* & 0.82 & 1.00 & 0.92 & 0.98 & $1.11^{\text {NS }}$ \\
& $(70.08)$ & $(59.17)$ & $(69.69)$ & $(65.33)$ & \\
\hline Total & 0.23 & 0.12 & 0.18 & 0.14 & $39.58^{* * *}$ \\
\hline & $(19.65)$ & $(7.10)$ & $(13.63)$ & $(9.33)$ & \\
\hline
\end{tabular}

Note: *Present fallow land: land that is not seeded for one or 2 growing season. Figures in parentheses represent Percentage to total. $* * *$ significant at $1 \%$ level, **significant at $5 \%$ level, NS - non significant

Table.2 Ownership of fixed assets under major farming systems in Kolar district

\begin{tabular}{|c|c|c|c|c|c|c|}
\hline Sl. No. & $\begin{array}{c}\text { Particulars } \\
\text { Average size of the } \\
\text { farm (Ha) }\end{array}$ & $\begin{array}{c}\mathbf{C}+\mathrm{Sh} \\
(\mathrm{n}=\mathbf{3 0}) \\
\mathbf{1 . 1 7}\end{array}$ & $\begin{array}{c}C+D+S \\
(n=30) \\
1.69\end{array}$ & $\begin{array}{c}C+D \\
(n=30) \\
1.32\end{array}$ & $\begin{array}{c}\mathbf{C}+\mathbf{D}+\mathbf{H} \\
(\mathbf{n}=\mathbf{3 0}) \\
\mathbf{1 . 5 0}\end{array}$ & $F$ value \\
\hline 1. & $\begin{array}{l}\text { Value of the land per } \\
\text { farm (Rs.) }\end{array}$ & $\begin{array}{l}2197500 \\
(99.58)\end{array}$ & $\begin{array}{l}24175000 \\
(98.41)\end{array}$ & $\begin{array}{l}2750000 \\
(94.02)\end{array}$ & $\begin{array}{l}3660000 \\
(85.66)\end{array}$ & $137.87^{* * *}$ \\
\hline 2. & $\begin{array}{l}\text { Value of the non land } \\
\text { farm asset (Rs.) }\end{array}$ & $\begin{array}{l}9186 \\
(0.41)\end{array}$ & $\begin{array}{l}389715 \\
(1.58)\end{array}$ & $\begin{array}{l}174752 \\
(5.97)\end{array}$ & $\begin{array}{l}612456 \\
(14.33)\end{array}$ & $286.96^{* * * *}$ \\
\hline 3. & $\begin{array}{l}\text { Total asset per farm } \\
\text { (Rs.) }\end{array}$ & $\begin{array}{l}2206686 \\
(100)\end{array}$ & $\begin{array}{l}24564715 \\
(100)\end{array}$ & $\begin{array}{l}2924752 \\
(100)\end{array}$ & $\begin{array}{l}4272456 \\
(100)\end{array}$ & $140.23^{* * *}$ \\
\hline 4. & $\begin{array}{l}\text { Total Asset per hectare } \\
\text { (Rs.) }\end{array}$ & 1298050 & 14535334 & 2215721 & 2848304 & $233.89^{* * *}$ \\
\hline
\end{tabular}

Note: Figures in parentheses represent Percentages to total. ${ }^{* * *}$ significant at $1 \%$ level, $* *$ significant at $5 \%$ level 
Table.3 Net income of farm households from various sources in Kolar district (Rupees/ annum)

\begin{tabular}{|c|c|c|c|c|c|}
\hline $\begin{array}{l}\text { Farming } \\
\text { systems }\end{array}$ & Crop & Livestock & Sericulture & $\begin{array}{l}\text { Non }{ }^{*} \text { farm } \\
\text { income }\end{array}$ & Total \\
\hline $\begin{array}{l}C+S h \\
(n=30)\end{array}$ & $\begin{array}{r}13256 \\
(12.07)\end{array}$ & $\begin{array}{r}74549 \\
(67.92)\end{array}$ & - & $\begin{array}{r}21950 \\
(19.99)\end{array}$ & $\begin{array}{r}109755 \\
(100)\end{array}$ \\
\hline $\begin{array}{l}C+D+S \\
(n=30)\end{array}$ & $\begin{array}{l}18332 \\
(4.86)\end{array}$ & $\begin{array}{l}136245 \\
(36.19)\end{array}$ & $\begin{array}{l}217316 \\
(57.72)\end{array}$ & $\begin{array}{r}4560 \\
(1.21)\end{array}$ & $\begin{array}{r}376453 \\
(100)\end{array}$ \\
\hline $\begin{array}{l}C+D \\
(n=30)\end{array}$ & $\begin{array}{l}16032 \\
(7.74)\end{array}$ & $\begin{array}{l}152184 \\
(73.55)\end{array}$ & - & $\begin{array}{r}38690 \\
(18.69)\end{array}$ & $\begin{array}{r}206906 \\
(100)\end{array}$ \\
\hline $\begin{array}{l}C+D+H^{1} \\
(n=30)\end{array}$ & $\begin{array}{l}108953 \\
(45.19)\end{array}$ & $\begin{array}{l}113152 \\
(46.93)\end{array}$ & - & $\begin{array}{l}18984 \\
(7.87)\end{array}$ & $\begin{array}{r}241089 \\
(100)\end{array}$ \\
\hline F value & $34.25^{* * *}$ & $631.50^{* * *}$ & $29.67^{* * *}$ & $208.30^{* * *}$ & \\
\hline
\end{tabular}

Note: Figures in parentheses represent Percentages to total*Non-farm income includes income earned by working in others field for wages, working in Governmental organizations, working in private organizations or through own enterprises like kirani shops etc. ***significant at $1 \%$ level, **significant at $5 \%$ level. NS - Non Significant. 1 - crop income include crop + horticulture income

Table.4 Quantity of food consumption and expenditure pattern of sample farm household per month in Kolar district

\begin{tabular}{|c|c|c|c|c|c|c|c|c|}
\hline \multirow[t]{2}{*}{ Food Item } & \multicolumn{2}{|c|}{$\begin{array}{c}\text { C+Sh } \\
(n=30)\end{array}$} & \multicolumn{2}{|c|}{$\begin{array}{c}C+D+S \\
(n=30)\end{array}$} & \multicolumn{2}{|c|}{$\begin{array}{c}\text { C+D } \\
(\mathbf{n}=30)\end{array}$} & \multicolumn{2}{|c|}{$\begin{array}{c}\mathrm{C}+\mathrm{D}+\mathrm{H} \\
(\mathrm{n}=30)\end{array}$} \\
\hline & Kg & Rs. & Kg & Rs & Kg & Rs & Kg & Rs \\
\hline Avg. family size (No) & \multicolumn{2}{|l|}{6} & \multicolumn{2}{|c|}{6} & \multicolumn{2}{|c|}{5} & \multicolumn{2}{|c|}{5} \\
\hline Cereals $(\mathrm{Kg})$ & $84(54.80)$ & 895 & $86(49)$ & 915 & $54(41)$ & 450 & $58(40)$ & 480 \\
\hline Pulses (Kg) & $\begin{array}{c}4.28 \\
(2.80)\end{array}$ & 234 & $\begin{array}{c}5.13 \\
(2.93)\end{array}$ & 330 & $\begin{array}{c}4.6 \\
(3.49)\end{array}$ & 248 & $\begin{array}{c}5.10 \\
(3.55)\end{array}$ & 310 \\
\hline Edible Oil (kg) & $\begin{array}{c}3.56 \\
(2.30)\end{array}$ & 221 & $\begin{array}{c}4.5 \\
(2.57)\end{array}$ & 290 & $\begin{array}{c}4.1 \\
(3.11)\end{array}$ & 230 & $\begin{array}{c}4.2 \\
(2.92)\end{array}$ & 270 \\
\hline VegeTables (Kg) & $\begin{array}{c}29.4 \\
(19.20)\end{array}$ & 553 & $\begin{array}{c}38 \\
(21.67)\end{array}$ & 650 & $\begin{array}{c}34.2 \\
(25.95)\end{array}$ & 590 & $\begin{array}{c}38 \\
(26.43)\end{array}$ & 580 \\
\hline Fruits and Nut (Kg) & $\begin{array}{c}5.00 \\
(3.30)\end{array}$ & 304 & $\begin{array}{c}6.12 \\
(3.49)\end{array}$ & 450 & $\begin{array}{c}5.0 \\
(3.79)\end{array}$ & 304 & $\begin{array}{c}6.14 \\
(4.27)\end{array}$ & 460 \\
\hline Egg and meat (kg) & $\begin{array}{c}5.6 \\
(3.70)\end{array}$ & 1360 & $\begin{array}{c}4.5 \\
(2.57)\end{array}$ & 1485 & $\begin{array}{c}3.8 \\
(2.88)\end{array}$ & 1254 & $\begin{array}{c}4.12 \\
(2.87)\end{array}$ & 1359 \\
\hline $\begin{array}{l}\text { Milk and Milk Products } \\
\text { (kg) }\end{array}$ & $\begin{array}{c}17 \\
(11.10)\end{array}$ & 374 & $\begin{array}{c}26 \\
(14.83)\end{array}$ & 528 & $\begin{array}{c}21 \\
(15.93)\end{array}$ & 462 & $\begin{array}{c}23 \\
(16)\end{array}$ & 483 \\
\hline Sugar \& Jaggery (kg) & $\begin{array}{c}4.51 \\
(2.90)\end{array}$ & 224 & $\begin{array}{c}5.12 \\
(2.92)\end{array}$ & 256 & $\begin{array}{c}5.1 \\
(3.87)\end{array}$ & 255 & $\begin{array}{c}5.2 \\
(3.62)\end{array}$ & 265 \\
\hline Others & & 120 & & 250 & & 200 & & 200 \\
\hline Total & $\begin{array}{c}153 \\
(100)\end{array}$ & 4885 & $\begin{array}{c}175 \\
(100)\end{array}$ & 5164 & $\begin{array}{c}132 \\
(100)\end{array}$ & 3993 & $\begin{array}{c}144 \\
(100)\end{array}$ & 4407 \\
\hline
\end{tabular}

(Note: 1 egg $=44 \mathrm{gm}$, Figures in parentheses represent Percentage to total) 
Table.4 Availability and accessibility to health services to farm households in Kolar district

\begin{tabular}{|l|l|c|c|c|c|}
\hline \multicolumn{1}{|c|}{ Particular } & Health center & $\begin{array}{c}\mathbf{C}+\mathbf{S h} \\
(\mathbf{n = 3 0})\end{array}$ & $\begin{array}{c}\mathbf{C}+\mathbf{D}+\mathbf{S} \\
(\mathbf{n = 3 0})\end{array}$ & $\begin{array}{c}\mathbf{C}+\mathbf{D} \\
(\mathbf{n = 3 0})\end{array}$ & $\begin{array}{c}\mathbf{C}+\mathbf{D}+\mathbf{H} \\
(\mathbf{n = 3 0})\end{array}$ \\
\hline \multirow{2}{*}{$\begin{array}{l}\text { Availability } \\
\text { (Per cent) }\end{array}$} & Primary & 25.12 & 33.33 & 65.12 & 42.25 \\
\hline $\begin{array}{l}\text { Accessibility } \\
\text { (Distance in km) }\end{array}$ & 24 Hours facility & 12.52 & 15.25 & 32.15 & 56.84 \\
\hline \multirow{2}{*}{$\begin{array}{l}\text { Accessibility } \\
\text { (Time in min) }\end{array}$} & Primary & 3.20 & 1.00 & 0.80 & 2.25 \\
\cline { 2 - 6 } & 24 Hours facility & 4.50 & 2.90 & 1.50 & 5.60 \\
\hline & Specialty hospital & 11.12 & 8.20 & 7.00 & 8.50 \\
\hline \multirow{2}{*}{ No. of farmers owning Yashaswini card } & Primary & 15.12 & 8.50 & 10.15 & 12.41 \\
\hline & 24 Hours facility & 18.20 & 15.50 & 15.12 & 16.42 \\
\cline { 2 - 6 } & Specialty hospital & 25.10 & 25.45 & 30.24 & 20.18 \\
\hline
\end{tabular}

Table.5 Household assets of farm household in Kolar district

\begin{tabular}{|c|c|c|c|c|c|c|c|c|}
\hline \multirow[t]{2}{*}{ Particular } & \multicolumn{2}{|c|}{$\begin{array}{l}\mathrm{C}+\mathrm{Sh} \\
(\mathbf{n}=\mathbf{3 0})\end{array}$} & \multicolumn{2}{|c|}{$\begin{array}{c}C+D+S \\
(n=30)\end{array}$} & \multicolumn{2}{|c|}{$\begin{array}{l}C+D \\
(\mathbf{n}=30)\end{array}$} & \multicolumn{2}{|c|}{$\begin{array}{c}C+D+H \\
(n=30)\end{array}$} \\
\hline & No. & $\begin{array}{l}\text { Value } \\
\text { (Rs.) }\end{array}$ & No. & Value (Rs.) & No. & $\begin{array}{l}\text { Value } \\
\text { (Rs.) }\end{array}$ & No. & $\begin{array}{c}\text { Value } \\
\text { (Rs.) }\end{array}$ \\
\hline $\begin{array}{l}\text { Type of House } \\
\text { 1. Kaccha }\end{array}$ & 7 & $\begin{array}{l}47210 \\
(12.51)\end{array}$ & 3 & $\begin{array}{l}55486 \\
(6.56)\end{array}$ & 5 & $\begin{array}{l}55789 \\
(8.17)\end{array}$ & 4 & $\begin{array}{l}56789 \\
(7.26)\end{array}$ \\
\hline 2. Semi Pakka & 22 & $\begin{array}{l}96412 \\
(25.55)\end{array}$ & 17 & $\begin{array}{l}194285 \\
(22.99)\end{array}$ & 20 & $\begin{array}{l}187590 \\
(27.48)\end{array}$ & 15 & $\begin{array}{l}210457 \\
(26.94)\end{array}$ \\
\hline 3. Pakka & 1 & $\begin{array}{l}184756 \\
(48.90)\end{array}$ & 10 & $\begin{array}{l}478953 \\
(56.68)\end{array}$ & 5 & $\begin{array}{l}365897 \\
(53.60)\end{array}$ & 11 & $\begin{array}{l}398745 \\
(51.04)\end{array}$ \\
\hline Toilet facility & 24 & $\begin{array}{l}11320 \\
(3.00)\end{array}$ & 29 & $\begin{array}{l}13475 \\
(1.59)\end{array}$ & 25 & $\begin{array}{l}11457 \\
(1.67)\end{array}$ & 30 & $\begin{array}{l}12987 \\
(1.66)\end{array}$ \\
\hline Coocking gas & 18 & $\begin{array}{l}5120 \\
(1.35)\end{array}$ & 28 & $\begin{array}{l}5240 \\
(0.62)\end{array}$ & 28 & $\begin{array}{l}5187 \\
(0.75)\end{array}$ & 30 & $\begin{array}{c}5421 \\
(0.69)\end{array}$ \\
\hline Value of the durable (Rs.) & & $\begin{array}{l}32410 \\
(8.59)\end{array}$ & & $\begin{array}{l}97425 \\
(11.53)\end{array}$ & & $\begin{array}{l}56714 \\
(8.30)\end{array}$ & & $\begin{array}{l}96784 \\
(12.38)\end{array}$ \\
\hline Total value (Rs.) & & $\begin{array}{c}377228 \\
(100)\end{array}$ & & $\begin{array}{c}844864 \\
(100)\end{array}$ & & $\begin{array}{c}682634 \\
(100)\end{array}$ & & $\begin{array}{c}781183 \\
(100)\end{array}$ \\
\hline
\end{tabular}

(Note: Figures in parentheses represent Percentage to total)

Table.6 Availability and accessibility to drinking water by the farm households in Mandya district

\begin{tabular}{|c|c|c|c|c|c|}
\hline Particular & Source & $\begin{array}{l}C+S h \\
(n=30)\end{array}$ & $\begin{array}{c}C+D+S \\
(n=30)\end{array}$ & $\begin{array}{l}C+D \\
(n=30)\end{array}$ & $\begin{array}{c}C+D+H \\
(n=30)\end{array}$ \\
\hline \multirow{3}{*}{$\begin{array}{l}\text { Availability } \\
\text { (Per cent) }\end{array}$} & Public source & 53.33 & 56.66 & 26.66 & 46.66 \\
\hline & Own bore well & 10 & 13.33 & 10 & 10 \\
\hline & Filter water & 36.66 & 30.00 & 63.33 & 43.33 \\
\hline \multirow{3}{*}{$\begin{array}{l}\text { Accessibility } \\
\text { (Distance in Mt.) }\end{array}$} & Public source & 64.33 & 57.89 & 80.76 & 65.75 \\
\hline & Own bore well & 1.50 & 1.45 & 1.10 & 0.55 \\
\hline & Filter water & 957 & 1142 & 1245 & 1124 \\
\hline \multirow{3}{*}{$\begin{array}{l}\text { Accessibility } \\
\text { (Time in min) }\end{array}$} & Public source & 2.15 & 1.43 & 3.25 & 2.23 \\
\hline & Own bore well & 0.30 & 0.41 & 0.25 & 0.15 \\
\hline & Filter water & 11.20 & 12.40 & 13.42 & 12.48 \\
\hline
\end{tabular}


Table.7 Availability and accessibility to drinking water by the farm households in Kolar district

\begin{tabular}{|c|c|c|c|c|c|}
\hline Particular & Source & $\begin{array}{l}\mathbf{C}+\mathbf{S h} \\
(\mathbf{n}=\mathbf{3 0})\end{array}$ & $\begin{array}{c}C+D+S \\
(n=30)\end{array}$ & $\begin{array}{l}C+D \\
(n=30)\end{array}$ & $\begin{array}{c}C+D+H \\
(n=30)\end{array}$ \\
\hline \multirow{3}{*}{$\begin{array}{l}\text { Availability } \\
\text { (Per cent) }\end{array}$} & Public source & 30.00 & 6.66 & 13.33 & 6.66 \\
\hline & Own bore well & - & 23.33 & - & 26.66 \\
\hline & Filter water & 70.00 & 70.00 & 86.66 & 66.66 \\
\hline \multirow{3}{*}{$\begin{array}{l}\text { Accessibility } \\
\text { (Distance in Mt.) }\end{array}$} & Public source & 221 & 248 & 252 & 124 \\
\hline & Own bore well & - & 1.12 & - & 2.12 \\
\hline & Filter water & 1300 & 1750 & 1325 & 2145 \\
\hline \multirow{3}{*}{$\begin{array}{l}\text { Accessibility } \\
\text { (Time in min) }\end{array}$} & Public source & 4.20 & 4.56 & 5.13 & 2.19 \\
\hline & Own bore well & - & 0.50 & - & 2.10 \\
\hline & Filter water & 12.13 & 13.45 & 13.4 & 15.42 \\
\hline
\end{tabular}

Table.8 Availability and accessibility of education institutes in Kolar district

\begin{tabular}{|l|l|c|c|c|c|}
\hline Particular & \multicolumn{1}{|c|}{ Source } & $\begin{array}{c}\mathbf{C}+\mathbf{S h} \\
(\mathbf{n = 3 0})\end{array}$ & $\begin{array}{c}\mathbf{C}+\mathbf{D}+\mathbf{S} \\
(\mathbf{n = 3 0})\end{array}$ & $\begin{array}{c}\mathbf{C}+\mathbf{D} \\
(\mathbf{n = 3 0})\end{array}$ & $\begin{array}{c}\mathbf{C}+\mathbf{D}+\mathbf{H} \\
(\mathbf{n = 3 0})\end{array}$ \\
\hline $\begin{array}{l}\text { Availability } \\
\text { (Per cent) }\end{array}$ & Primary School & 80 & 95 & 83.33 & 93.33 \\
\hline $\begin{array}{l}\text { Accessibility } \\
\text { (Distance in Km.) }\end{array}$ & High School & 36.66 & 64.53 & 58.45 & 60.00 \\
\hline & Himary School & 0.90 & 0.45 & 0.82 & 0.60 \\
\hline & High School & 3.12 & 1.98 & 3.2 & 1.84 \\
\hline $\begin{array}{l}\text { Accessibility } \\
\text { (Time in min) }\end{array}$ & Primary School & 6.2 & 3 & 6 & 9.00 \\
\cline { 2 - 6 } & High School & 14.2 & 8 & 12 & 6.2 \\
\cline { 2 - 6 } & College and Degree & 28 & 23.4 & 26 & 16 \\
\hline
\end{tabular}

Table.9 Social participation status of the farm households in Kolar district (in number)

\begin{tabular}{|c|c|c|c|c|c|}
\hline SI No. & Particulars & $\begin{array}{l}C+S h \\
(n=30)\end{array}$ & $\begin{array}{c}C+D+S \\
(n=30)\end{array}$ & $\begin{array}{c}C+D \\
(n=30)\end{array}$ & $\begin{array}{c}C+D+H \\
(n=30)\end{array}$ \\
\hline 1. & Member in Gram Panchayath & $1.00(3.33)$ & $\begin{array}{c}3 \\
(10.00)\end{array}$ & $\begin{array}{c}1 \\
(3.33)\end{array}$ & $\begin{array}{c}3 \\
(10.00)\end{array}$ \\
\hline 2. & Member in Taluk Panchayath & - & - & - & $\begin{array}{c}1 \\
(3.33)\end{array}$ \\
\hline 3. & $\begin{array}{l}\text { Member in Milk Producers Co- } \\
\text { operative Society }\end{array}$ & $\begin{array}{c}2 \\
(6.66)\end{array}$ & $\begin{array}{c}29 \\
(96.66)\end{array}$ & $\begin{array}{c}30 \\
(100.00)\end{array}$ & $\begin{array}{c}30 \\
(100.00)\end{array}$ \\
\hline 4. & Member in SH Group & $\begin{array}{c}27 \\
(90.00)\end{array}$ & $\begin{array}{c}22 \\
(73.33)\end{array}$ & $\begin{array}{c}26 \\
(86.66)\end{array}$ & $\begin{array}{c}22 \\
(73.33)\end{array}$ \\
\hline 5. & Television (No) & $23(76.66)$ & $\begin{array}{c}28 \\
(93.33)\end{array}$ & $\begin{array}{c}27 \\
(90.00)\end{array}$ & $\begin{array}{c}28 \\
(93.33)\end{array}$ \\
\hline 6. & Phone (No.) & $38(126.66)$ & $\begin{array}{c}55 \\
(183.33)\end{array}$ & $\begin{array}{c}42 \\
(140.00)\end{array}$ & $\begin{array}{c}52 \\
(173.33)\end{array}$ \\
\hline
\end{tabular}

(Note: Figures in parentheses represent Percentage to total number of sample) 
Table.10 Composite livelihood security index of sample farm households of Kolar district

\begin{tabular}{|l|c|c|c|c|c|}
\hline Particulars (Index) & $\begin{array}{c}\mathbf{C}+\mathbf{S h} \\
(\mathbf{n = 3 0})\end{array}$ & $\begin{array}{c}\mathbf{C}+\mathbf{D}+\mathbf{S} \\
(\mathbf{n = 3 0})\end{array}$ & $\begin{array}{c}\mathbf{C}+\mathbf{D} \\
(\mathbf{n}=\mathbf{3 0})\end{array}$ & $\begin{array}{c}\mathbf{C}+\mathbf{D}+\mathbf{H} \\
(\mathbf{n = 3 0})\end{array}$ & F value \\
\hline Economic security & 0.23 & 0.48 & 0.38 & 0.42 & $24.50^{* * *}$ \\
\hline Food security & 0.38 & 0.42 & 0.37 & 0.47 & $4.56^{* * *}$ \\
\hline Health security & 0.24 & 0.28 & 0.40 & 0.45 & $14.51^{* * * *}$ \\
\hline Habitat security & 0.34 & 0.51 & 0.45 & 0.49 & $21.53^{* * *}$ \\
\hline Educational security & 0.42 & 0.50 & 0.49 & 0.57 & $3.46^{* * *}$ \\
\hline Social participation security & 0.32 & 0.55 & 0.48 & 0.59 & $39.41^{* * *}$ \\
\hline Over all livelihood security & 0.322 & 0.44 & 0.42 & 0.49 & $14.23^{* * *}$ \\
\hline
\end{tabular}

Note: *** significant at $1 \%$ level, ** significant at $5 \%$ level

Table 4 reveals that Percentage of availability of primary health center was highest in $\mathrm{C}+\mathrm{D}$ (65.12 per cent) farming system households with in the radius of $0.8 \mathrm{~km}$ and it takes average of ten minutes to reach followed by $\mathrm{C}$ $+\mathrm{D}+\mathrm{H}$ farming system. There is no specialty Hospital facility within the radius of $20 \mathrm{~km}$ in any one of the farm households in both districts. Highest number of Yashaswini card holders found in $\mathrm{C}+\mathrm{D}+\mathrm{H}$ farm households than the other farming systems in the districts

\section{Habitat Security}

Habitat of the household is another factor which influences the livelihood of household. Type of house, toilet facility, availability of cooking gas, drinking water and value of the durables (television, phone, fans, almirahs, motor cycle, fridge etc.) are the parameters which influences habitat security. Table 5, 6 and 7 indicate that, total value of household assets found higher in C + D + S (Rs. 844864) farm households followed by $\mathrm{C}+\mathrm{D}+\mathrm{H}$ farming system. Government programs like MGNAREGA and Ujjwala Yojana have benefited more than 60 percent of farm households by providing toilet facility and cooking gas facility in both the districts. With respect to the availability and accessibility of the drinking water more than 66 per cent of the farm households use filtered water as public source of drinking water as the ground water extracted from deeper layer has higher salt load. Only 20 percent of the farm households depend on their own bore well for drinking water in $\mathrm{C}+\mathrm{D}+\mathrm{S}$ and $\mathrm{C}+\mathrm{D}+\mathrm{H}$ farming system.

\section{Education Security}

Education is the most important aspect of life. Availability and accessibility of education institutes to farm households were taken for calculation of education security. Table 8 reveals that more than 80 Per cent of respondents have primary schools available within a radius of $1 \mathrm{~km}$. However, none of the households in any of the farming systems have college and degree in the vicinity of $10 \mathrm{~km}$ in the districts. That means students from these farming system households have to travel more than 20 minutes, which accounts for expenditure on education.

\section{Social participation Security}

Social network is nothing but the level of participation by the households in organizations like Panchayats, Milk cooperatives and Self Help Groups, access to elements like phone and television is another factor which determines social network status of households. The Table 9 reveals that participation in organizations like Panchayath (3) and co-operatives (30) was found higher 
in $\mathrm{C}+\mathrm{D}+\mathrm{H}$ farming system in Kolar district and also 93.33 per cent of households had access to television in $\mathrm{C}+\mathrm{D}+\mathrm{H}$ farming system followed by $\mathrm{C}+\mathrm{D}+\mathrm{S}$ farming system with assessment of 86.66 Per cent and 93.33 respectively. Interestingly participation in SHGs was found to be highest (90 Per cent) among $\mathrm{C}+$ Sh farming system households in the district due to small savings and need of credit to the farm households and usage of phone in all the farming systems in the district is more than 100 Per cent.

\section{Composite livelihood security index for farm households in the study area}

Livelihood security index includes subcomponents of food security, economic security, education security, health security, habitat security and social participation securities. From the Table 10 indicate that the composite livelihood security index was found to be lowest (0.35) in $\mathrm{C}+\mathrm{Sh}$ farming system households. It is due to less landholding, less annual income, least participation in social activities and availability and accessibility of the education institution. The composite livelihood security index was found to be higher (0.49) in C+ D + $\mathrm{H}$ farming system households as they have good land holdings i.e., 1.85 and 1.50 ha, good irrigation facilities, labour force annual net income, accessibility and availability of education, health institution and also good social participation. $F$ value indicates, a significant difference in all the subcomponents included in calculation. Composite livelihood security revealed that $\mathrm{C}$ + Sh farm households were less secured than the $\mathrm{C}+\mathrm{D}+\mathrm{S}, \mathrm{C}+\mathrm{D}$ and $\mathrm{C}+\mathrm{D}+\mathrm{H}$ farming system households in the district.

In conclusion the understanding the livelihood security of the farm households gives a holistic picture of economics, food, health, habitat, education and social participation security of the households. In this motive, present research work is done to understand the overall livelihood security of farmers who practice the various farming systems like Crop + Sheep, Crop +Dairy, Crop + Dairy + Sericulture and Crop + Dairy + Horticulture in Eastern Dry Zone of Karnataka. Based on the result of the study, dietary pattern of households in all the groups was mainly cereal based which needs to be reported to Government and encourage it to take initiation to distribute pulses through Public distribution system and there is a need for providing the basic facilities like education institution, hospitals, drinking water facility in the vicinity to increase the overall livelihood security of the farming households. Dairy and sericulture enterprises are complementary to each other and provide stability to sustain farm income. Subject to technical feasibility, promotion of these enterprises to strengthen livelihood security

\section{References}

Aktershaheen and Rahman, S. 2012. investigating livelihood security in poor settlements in Bangladesh. Paper presented In: Annual Conference of Agricultural Economics Society, University of Warwick, United Kingdom.

Harishkumar, H. V., Umesh, K. B., Satish Kumar, M. and Murali, D. N. 2016. Livelihood security of farm households under different farming systems in Kolar district of Karnataka - An economic analysis. International $J$. Agric., Environ. Biotechnology., 9 (2): 313-322.

Ijarotimi, O.S. and Oyeneyin, O.O. 2005. Effect of economic restructuring on household food security and nutritional status of Nigerian children. J. Food, Agric. Environ., 3(2): 27-32.

Maxwell, S. and Frankenberger, T., 
1992, Household Food Security: Concepts, Indicators, and Measurements: A technical review. Food forum, No.34 Washington, DC, USA.

Norman, D.W., 1978, Farming systems research to improve the livelihood of small farmers. American J. Agric. Econ., 60: 813-818.

Sachin, T. N., 2012, Economics of farming systems in northern transitional zone of Karnataka. Ph.D (Agri.) Thesis(Unpub.), Univ. of Agri. Sci., Dharwad.

Sakamma, S., 2013, Women empowerment and livelihood security of farm households: An economic analysis. Ph.D Thesis, University of Agricultural Science, GKVK,
Bengaluru.

Shyamali, H.W and Saini, A.S., 2013, Livelihood security of women in hills: A comparative study of India and Srilanka. Ind. J. Agric. Econ., 65(6): 710-721.

Venu, B. N., Umesh, K. B., and Gajanana, T. M., 2018, Livelihood security of agricultural labour households in rainfed region of north- Karnataka An economic analysis. Ind. J. Agric. Res., 52(5): 463-471.

Zainab, N., 2010, A study on economic analysis of integrated farming systems in central dry zone of Karnataka. $M$. Sc. (Agri.) Thesis(Unpub.), Univ. of Agric. Sci., Bangaluru. www.indiastat.com

\section{How to cite this article:}

Bhavya, A. P., B. C Ashwini and Umesh, K. B. 2020. Livelihood Security of Farm Households in Eastern Dry Zone of Karnataka - An Economic Analysis. Int.J.Curr.Microbiol.App.Sci. 9(08): 2951-2960. doi: https://doi.org/10.20546/ijcmas.2020.908.331 